\title{
Analyst
}

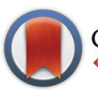

CrossMark

\& click for updates

Cite this: Analyst, 2016, 141, 731

Received 15th October 2015, Accepted 24th November 2015

DOI: 10.1039/c5an02129d

www.rsc.org/analyst

\section{Micro-scale spatially offset Raman spectroscopy for non-invasive subsurface analysis of turbid materials}

\begin{abstract}
P. Matousek, ${ }^{* a}$ C. Conti, ${ }^{\star b}$ M. Realini ${ }^{b}$ and C. Colombo ${ }^{b}$
This article reviews a very recent field of noninvasive analysis of turbid media using micro-scale Spatially Offset Raman Spectroscopy - micro-SORS. The technique combines conventional SORS with microscopy concepts and represents a new imaging modality in Raman microscopy. Micro-SORS facilitates analytical capability for investigating non-destructively the chemical composition of subsurface, micrometerscale-thick diffusely scattering layers at depths more than an order of magnitude larger than those accessible with the depth resolving power of conventional confocal Raman microscopy. Potential application areas include nondestructive subsurface analysis of painted layers in cultural heritage, characterization of stratified polymer systems, analysis of layered biological samples or forensic analysis. The article discusses the basic principles of the technique, its variants and outlines emerging applications in this rapidly evolving field.
\end{abstract}

\section{Introduction}

Confocal Raman microscopy is a powerful analytical technique which has found its widespread use across a vast number of analytical areas ranging from biomedicine and biology to

${ }^{a}$ Central Laser Facility, Research Complex at Harwell, STFC Rutherford Appleton Laboratory, Harwell, Oxford, OX11 OQX, UK. E-mail: pavel.matousek@stfc.ac.uk

${ }^{b}$ Consiglio Nazionale delle Ricerche, Istituto per la Conservazione e la Valorizzazione dei Beni Culturali (ICVBC), Via Cozzi 53, 20125 Milano, Italy.

E-mail: c.conti@icvbc.cnr.it

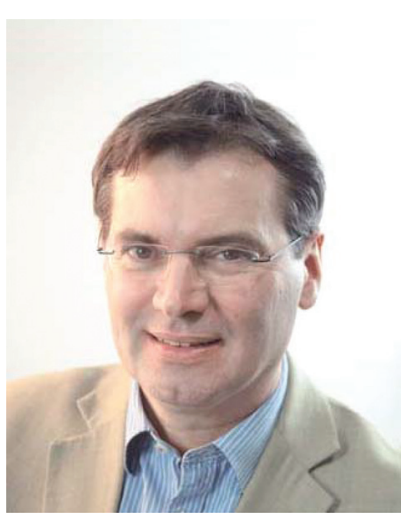

P. Matousek
Prof. Pavel Matousek has worked at the Rutherford Appleton Laboratory in the area of vibrational spectroscopy for over 24 years. He pioneered the concepts of Kerr gated Raman spectroscopy and Spatially Offset Raman Spectroscopy (SORS). Pavel has published around 200 peerreviewed articles and filed 10 patents. His honours include the premier Royal Academy of Engineering's $2014 \quad$ MacRobert Award, 2009 Charles Mann Award and the 2002 \& 2006 Meggers Awards. Pavel is a Fellow of the Royal Society of Chemistry, a Fellow of the Society for Applied Spectroscopy, a Senior Fellow of STFC, an honorary professor at the University College London and a founding Director of Cobalt Light Systems. forensics, semiconductors, pharmaceutics, geology and materials research and manufacture to name only some. ${ }^{1}$ Although the technique is applicable to non-invasive probing of transparent or semi-transparent materials, its applicability to scanning indepth within highly turbid (diffusely scattering) samples is severely limited due to its inability to provide direct optical imaging within samples at depths where photon diffusion propagation mechanisms become dominant. With such highly turbid matrices, often encountered with paint, coatings or biological samples, the depth penetration of the technique can be restricted to several micrometres or tens of micrometres in

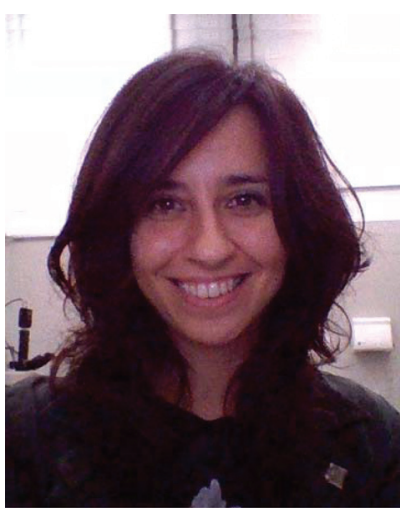

C. Conti
Dr Claudia Conti, researcher at the Institute for the Conservation and Valorization of Cultural Heritage (ICVBC) of the National Research Council (CNR), obtained her PhD degree in Materials Engineering from the Milan Polytechnic in 2010. Through her PhD and research at ICVBC she established an expertise in the area of advanced applications of vibrational spectroscopy to the analysis of materials, in particular in Cultural Heritage. She supervises the Raman Laboratory at ICVBC, started by her after her PhD. In 2014 she was a visiting scientist at the STFC Rutherford Appleton Laboratory, under CNR's Short Term Mobility program. She has published around 35 peerreviewed papers in leading journals in respective fields. 
some cases. Many analytical problems requiring deeper noninvasive probing in these matrices are therefore not addressable by this conventional approach. This limitation of confocal Raman microscopy often necessitates resorting to cross sectional analysis to retrieve chemical information from deeper layers. The cross sectional analysis is, however, invasive and often highly undesirable or in some situations not permissible: examples of relevant matrices include artworks in cultural heritage such as panel, canvas and mural paintings, painted statues or other decorated objects. Painted layers are highly turbid and typically only a few tens of micrometres thick and often spread in multiple stratigraphy.,3 Other application examples include analysis of stratifications within polymers, forensic applications, paper or biological samples such as seeds, both in research and in quality control.

Recently a new concept in Raman microscopy capable of overcoming the limit of accessible depths has emerged micro-SORS. The concept builds on earlier advances of a related technique developed in recent years, Spatially Offset Raman Spectroscopy (SORS). ${ }^{4,5}$ Micro-SORS has distinct advantages over both conventional SORS and confocal Raman microscopy. First, Micro-SORS is able to analyse turbid layers beyond the reach of confocal Raman microscopy. Second, micro-SORS is able to resolve thin separate layers unlike conventional SORS which would only sense them as a composite super layer (i.e. being insensitive to its internal structure). In general, SORS principles are related to concepts used in NIR absorption tomography ${ }^{6-8}$ and fluorescence spectroscopy $^{9-12}$ which can also utilise the detection of optical signals separated from the point of illumination. However, conceptually there are some differences; for example, with NIR the detection of signal is performed at the same wavelengths as those injected into the sample posing difficulties for example in suppressing specular type reflections whilst in fluorescence spectroscopy the detection wavelengths are shifted away from the laser illumination (as with SORS) and the laser illumination wavelength has to be within an absorption band of detected species to induce the fluorescence in the first place posing restrictions on the choice of wavelengths - unlike with SORS where no absorption is required for the induction of Raman process. The penetration depths of the NIR and fluorescence techniques are however often higher than those of SORS as they deal typically with significantly stronger signals. On the other hand, the chemical content derived from SORS measurements is often considerably higher than that available from its counterparts defining its distinct application niches.

A conventional SORS approach relies on spatially separating the Raman collection zone from the laser illumination zone on the sample surface. Such spatially offset Raman spectra contain varying relative contributions from different sample depths. ${ }^{5}$ This is a consequence of the photons migrating to spatially separated zones near the surface having a higher likelihood of being lost at the sample-to-air interface than photons migrating through deeper zones. Statistically the mean photon penetration depth increases with increasing spatial offset. Such data sets enable the retrieval of Raman spectra of sublayers within stratified turbid matrixes. For a two layer sample at least two SORS spectra acquired at different spatial offsets are required to recover the Raman spectra of individual layers. One such spectrum would typically be a zero spatially offset spectrum (equivalent to a conventional backscattering Raman spectrum) and one obtained at a non-zero spatial offset. The numerical recovery of spectra from individual layers is achieved by a scaled subtraction of the two spectra

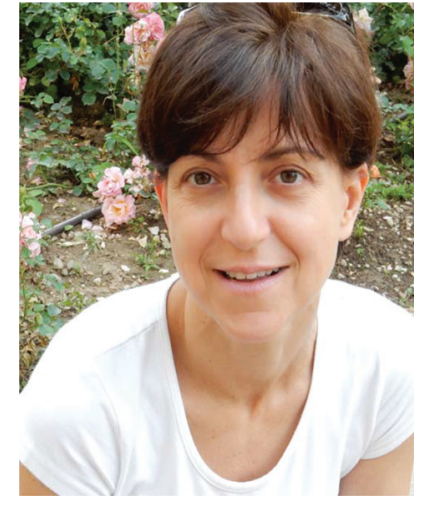

C. Colombo
Dr Chiara Colombo has been a researcher in Conservation Science at the Institute for the Conservation and Valorization of Cultural Heritage (ICVBC) of the National Research Council (CNR) for over 14 years. Her research line focuses on the characterization of natural and artificial stones and their decay processes; the study of inorganic conservation treatments in carbonatic matrices and the evaluation of new analytical methods to investigate their penetration and effectiveness; the monitoring of natural and artificial stone surfaces, with or without polychrome finishing using non-invasive and non-destructive analytical techniques. Chiara has published around 120 peer-reviewed articles. the Politecnico di Milano in Preservation of the Architectural tage. His research activity yielded 115 peer-reviewed papers and 106 technical reports. 
from each other with the multiplication factor adjusted to just cancel the contribution of the undesired layer leaving behind only the contributions from the layer one is recovering.

\section{Micro-SORS technique}

In its very essence, micro-SORS concept constitutes a merger of SORS and microscopy concepts. The most basic variant of micro-SORS technique is defocusing micro-SORS ${ }^{2}$ derived from early research into defocusing SORS. ${ }^{13-15}$ Although this is not the most effective concept available, ${ }^{16}$ as it does not involve fully separated laser illumination and Raman collection zones leading to lower contrast between individual layers derived from SORS, it is undoubtedly the most practical due to its extreme simplicity and suitability for use with existing conventional Raman microscopes without any modifications. Consequently, most research to date in micro-SORS has been based around this modality.

The concept of defocusing micro-SORS is illustrated in Fig. 1 for a two layer turbid system. The basic measurement relies on collecting at least two Raman spectra using a conventional Raman microscope. The first spectrum is acquired with the sample in a standard 'imaged' position where conventional Raman microscopy would traditionally be performed to analyse the surface of the sample. Then, a second measurement is taken with the sample moved away from the microscope objective by a 'defocusing distance $\Delta z$ ', to a so called 'defocused' position. (The reader should note that standard depth profiling in conventional confocal Raman microscopy would entail the opposite sample movement, i.e. towards the objective.) The effect of the sample displacement is the enlargement ('defocusing') of both the laser illumination and Raman collection zones on the sample surface. The larger the defocusing distance the greater the enlargement of both the laser illumination and Raman collection zones on the sample surface. This position is also reminiscent of global illumination Raman microscopy. The key difference is the presence of a step in micro-SORS Raman analysis where the acquired 'imaged' and 'defocused' spectra are compared for their spectral content (see Fig. 1 and 2). The 'imaged' position measurement yields a Raman spectrum dominated by the surface layer $^{17}$ and would correspond conceptually to a zero-spatially offset measurement in conventional SORS analysis. The measurement carried out in the 'defocused' position produces a Raman spectrum which has a significantly higher degree of Raman contribution from sublayers, in analogy with a nonzero spatially offset spectrum acquired in a conventional SORS measurement. ${ }^{13}$ The relative intensity change within spectral subcomponents signifies the presence of multiple layers in the sample. This is illustrated in Fig. 2 where a two-layer plastic system is measured with micro-SORS; polytetrafluoroethylene (PTFE) on top of polypropylene (PP). With increasing defocusing distance $\Delta z$ the relative Raman contribution of the sublayer (PP) increases over the surface layer Raman component (PTFE).

A simple mathematical manipulation of the 'imaged' and 'defocused' spectra can then be used to retrieve a pure Raman spectrum of sublayer. This process involves a scaled subtraction of the 'imaged' spectrum from the 'defocused' spectrum cancelling the contribution of the top layer (see Fig. 3). The pure Raman spectrum of the top layer can be obtained in analogy by a reverse process - a scaled subtraction of the 'defocused' spectrum away from the 'imaged' spectrum cancelling any features due to the sublayer. A pure spectrum from the top layer may be desirable, for example, in situations where the top layer is thin and conventional Raman microscopy renders a spectrum which is a mixture of the Raman spectra of the top layer and sublayer. In this situation a pure spectrum of the top layer could not be recovered from such a single Raman measurement without a priori knowledge of the composition and position of at least one of the layers. In the concerned experimental demonstration good correspondence between the spectra recovered by the scaling procedure and reference spectra has been achieved apart from residual noise which is naturally higher for the spectrum of the sublayer (see Fig. 3). It

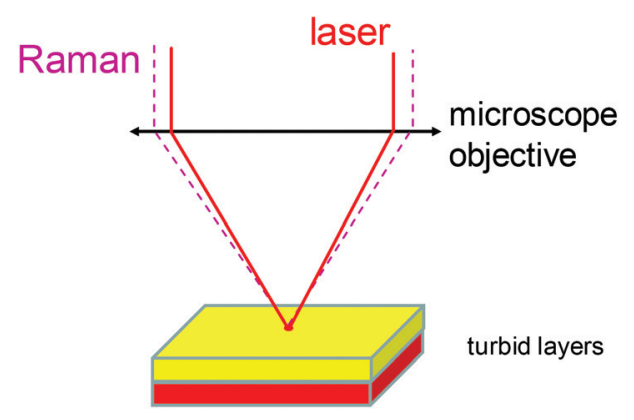

a) Imaged

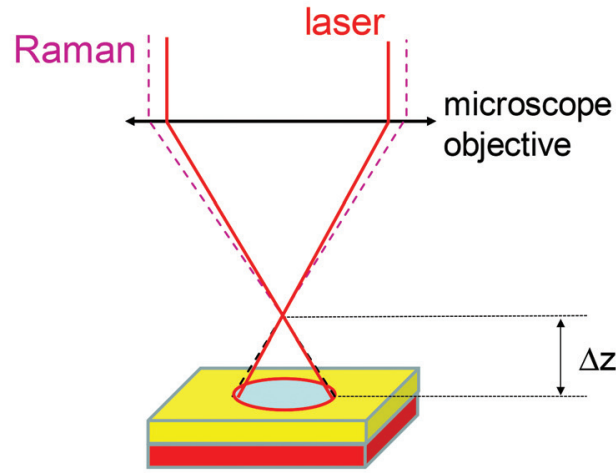

b) Defocused

Fig. 1 Schematic diagram of micro-SORS analysis. The first measurement is taken in a standard 'imaged' position (a) and the second in a 'defocused' position (b) implemented by displacing $(\Delta z)$ the sample away from the microscope objective. 

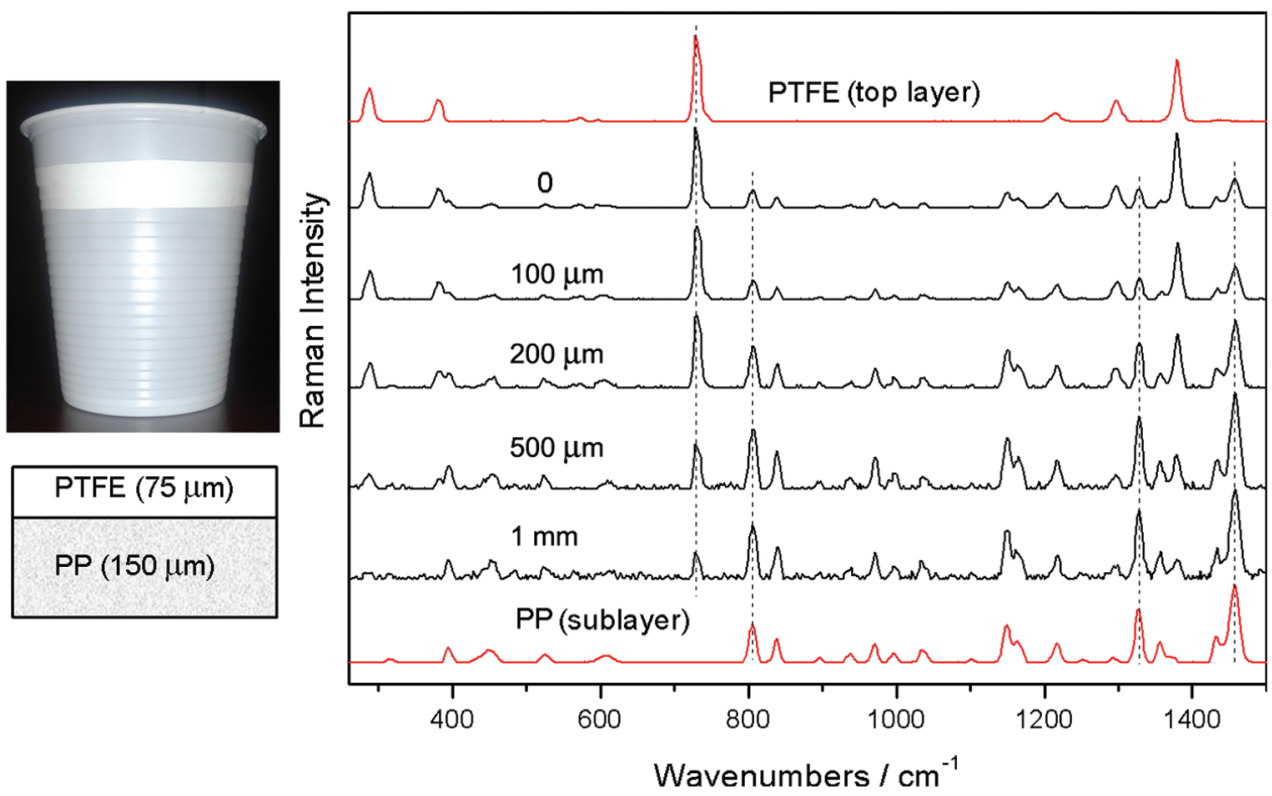

Fig. 2 Micro-SORS measurement of a two layer diffusely scattering polymer system (the top layer was a polytetrafluoroethylene (PTFE) tape placed over a sublayer of a plastic drinking cup made of polypropylene (PP)); image and stratigraphic scheme of the sample (left); defocused Raman spectra shown for different distances $\Delta z$ from the "imaged" plane $(\Delta z=0)$ indicated next to each spectrum (right). The spectra are offset for clarity. Note the line markers are for guidance to emphasize the changing relative intensity of polytetrafluoroethylene (PTFE) and polypropylene (PP) with the defocusing distance; the reference spectra (the top and bottom) are acquired on the sample using conventional Raman spectroscopy. ${ }^{17}$

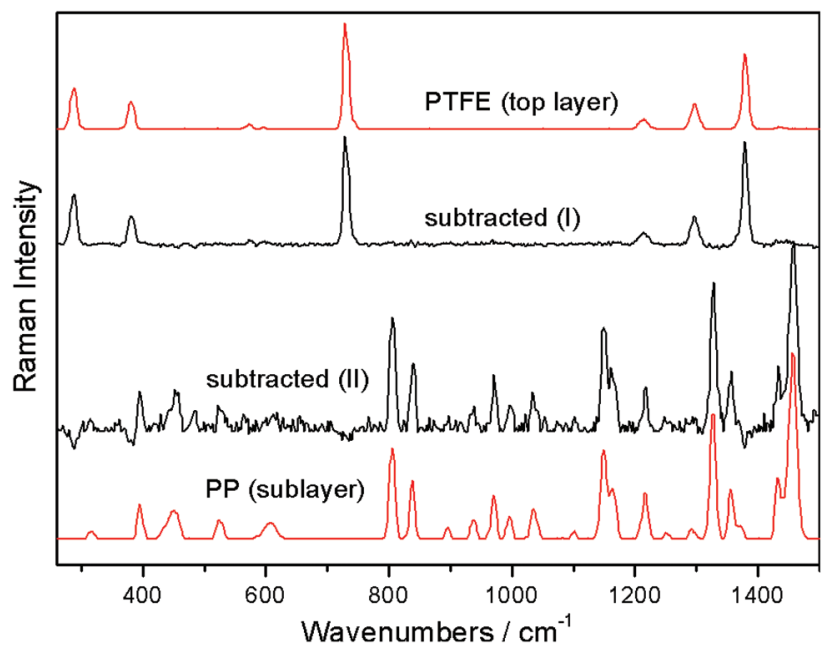

Fig. 3 Numerical recovery of pure Raman spectra of individual layers from the micro-SORS measurements carried out on the two-layer polymeric system depicted in Fig. 2: (I) recovered top layer spectrum and (II) recovered sublayer spectrum. Reference spectra are shown in top and bottom positions. ${ }^{17}$

should be noted that the scaling factors do not vary linearly with the displacement $\Delta z$. The dependence is highly nonlinear with a steeper initial dependence followed by a plateau for larger displacements $\Delta z^{2}$

The concept of scaled subtraction was first applied to confocal Raman microscopy with transparent samples by the Morris group. ${ }^{18,19}$ In these measurements the Raman spectra of overlapped images within a transparent sample were deconvolved from spectra obtained at different sample depths. This provided a stack of well-resolved Raman images describing the three-dimensional topology of the sample. Although microSORS deals with turbid samples and relies on photon diffusion rather than direct imaging the underlying principles are otherwise similar.

As the Raman signal is typically stronger at the 'imaged' position than in the 'defocused' position, this reduction of the signal in the 'defocused' position can be compensated either using longer acquisition times or higher laser powers. For the latter, the risk of damaging the sample due to excess heating is mitigated by an enlarged laser illumination zone on the sample surface.

At first glance, it may not be immediately obvious how SORS spatial offset mechanism relates to the micro-SORS measurements as both the laser and Raman collection zones remain overlapped in both the 'imaged' and 'defocused' positions. The situation becomes clearer when we consider the process at the individual photon level. Each detected Raman photon can be traced theoretically back to its originating laser photon. With the enlarged illumination and collection zones, the exit point of the Raman photon can be spatially separated at the sample surface from the entry point of its originating laser photon. This separation can range from none to a maximum possible value determined by the diameter of the laser illumination/Raman collection zone. That is when the laser photon excitation and Raman photon collection points at the sample surface are exactly at the opposite edges of the illumination/Raman collection zone. The entire ensemble of 
Raman photons detected in this way will therefore have an associated distribution of spatial offsets. Consequently, there will be a net non-zero SORS spatial offset present in the overall Raman spectrum acquired in the 'defocused' position. The magnitude of this net spatial offset can be controlled by the overall degree of defocusing introduced, i.e. the diameter of the illumination/Raman collection zone - the larger defocusing leading to a larger effective spatial offset. In turn, the 'imaged' collection yields a near zero spatially offset Raman spectrum as the photons can have only near-zero spatial offset due to their excitation and collection zones being confined to effectively a single, overlapped spot.

The theoretical foundations of micro-SORS were formed by Di et $a .^{20}$ and Matousek et al. ${ }^{21}$ using Monte Carlo simulations in analogy with earlier work on conventional SORS. ${ }^{22}$ The latter micro-SORS study ${ }^{21}$ reproduced in Fig. 4 concluded that penetration depths larger than an order of magnitude or two are possible with defocusing micro-SORS compared with the accessible depths achievable with conventional confocal Raman microscopy. The thickness of the two layers was maintained the same in the simulations and in a range of $10-40 \mu \mathrm{m}$ for the studied thin-layer configurations. In particular, Fig. 4 shows the enhancement of the relative intensity of Raman signal from the lower of the two thin layers over the top thin layer for a situation with low absorption and a photon transport length of $1 \mu \mathrm{m}$. The relative intensity of Raman signal from the bottom layer over top layer ('enhancement factor') is plotted normalised to the ratio of the same intensities obtained at $5 \mu \mathrm{m}$ illumination/collection diameter. The plot evidences that the enhancement factor improves rapidly within the defocusing range of 5 to $200 \mu \mathrm{m}$. Subsequent improvement above $200 \mu \mathrm{m}$ is relatively small with dependence plateauing. The enhancement factor is also found to be higher for thicker layers. Fig. 4b shows the overall dependence of the depth of origin of Raman photons on the degree of defocusing. A similar trend is observed here too; a sharp rise is present up to $200 \mu \mathrm{m}$ diameters followed by a plateau region.
The depth range down to which $50 \%$ of photons originate from (median depth) increases from $1.7 \mu \mathrm{m}$ to $9 \mu \mathrm{m}$ when going from the imaged to the largest defocused diameter (this in turn also means that $50 \%$ of photons originate from greater depths than the median value). The $90 \%$ quantile indicates the depth range down to which $90 \%$ of Raman photons originate from. It increases from $7.4 \mu \mathrm{m}$ to $26 \mu \mathrm{m}$ over the same range. The accessible depths can be contrasted with those achievable with depth accessing capability of conventional confocal Raman microscopy which, in this situation, would be approximately restricted to a photon transport length - in this case therefore to only $\sim 1 \mu \mathrm{m}$. This is because the photon directions are fully randomised upon this propagation distance and direct imaging of subsurface sample regions on which confocal Raman microscopy crucially depends is lost. In sharp contrast the above results of Monte Carlo simulations indicate that under the same conditions using the largest defocusing $(800 \mu \mathrm{m})$ the micro-SORS technique is capable of deriving $10 \%$ of its signal from depths larger than $26 \mu \mathrm{m}$, and a smaller fraction from yet greater depths - $1 \%$ of Raman signal was evaluated to originate from depths greater than $45 \mu \mathrm{m}$ in the concerned case. That is from 45-times greater depths than those accessible with conventional confocal Raman microscopy depth resolving power under the same situation. It should be noted that micro-SORS spatial resolution in general is considerably lower than that of confocal Raman microscopy and as such it should only be considered as an extension of confocal Raman microscopy capability at depths where conventional methods are inapplicable.

In general, the limitations of the micro-SORS technique include inapplicability to highly absorbing or highly fluorescing samples, extremely thin sublayers and compounds with low Raman cross sections at the subsurface position. In addition, samples possessing very high heterogeneity horizontally (at 90 degrees to the $z$-axis), within the layers are also challenging for the analysis without recourse to a more complex data acquisition methodology. ${ }^{3}$
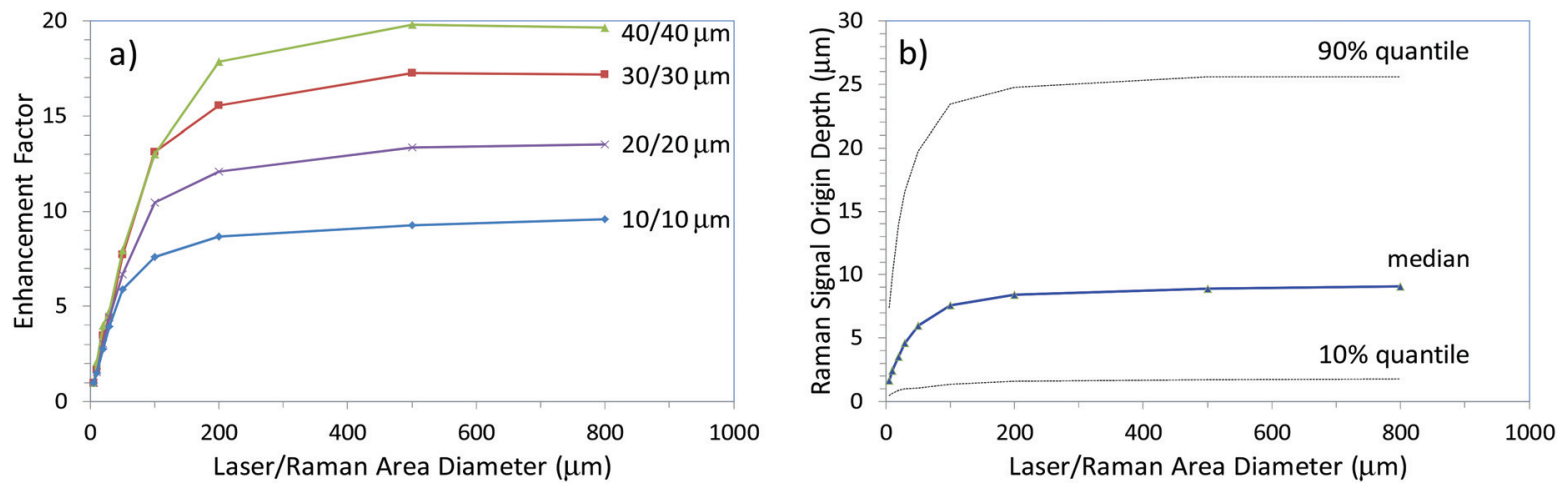

Fig. 4 The results of Monte Carlo simulations depicting (a) the enhancement of sublayer signal (normalised) in defocusing micro-SORS measurements for different layer thicknesses (indicated next to curves), (b) the penetration depth into medium expressed as median, $10 \%$ quantile and $90 \%$ quantile depth ranges down to which the corresponding fractions of Raman signals originate from as a function of laser illumination and Raman collection area diameters. The calculations were performed for a low absorption situation $\left(\alpha=0.23 \mathrm{~mm}^{-1}\right)$ and a photon transport length of $1 \mu \mathrm{m} .{ }^{21}$ 
The degree of relative performance of the defocusing variant of micro-SORS versus more advanced versions including also complete separation of the illumination and collection zones was analysed both theoretically and experimentally by Conti et al. ${ }^{16}$ The experimental study concluded that the full separation of laser illumination and Raman collection zones, in line with theoretical expectations, leads to much larger enhancement of the sublayer in comparison with the surface layer than with the basic defocusing micro-SORS; by an order of magnitude for the analysed situation comprising two thin turbid layers on top of an extended third turbid layer. Fig. 5 illustrates the experimental results. The measurements were performed on a 3 layer pigment system: red at the top location ( $\mathrm{R}$ - red ochre), white in the middle ( $\mathrm{W}$ - titanium white) and yellow at the bottom ( $\mathrm{Y}$ - bismuth vanadate). The micro-SORS spectra obtained using the method with fully separated illumination and collection points (SO) are shown in Fig. 5a. The '0' spatial offset spectrum represents a conventional Raman spectrum and is dominated by Raman bands associated with the top layer (R). The Raman bands of the second layer (W) are also clearly visible. No Raman spectra of the substrate layer are seen at this initial position. The gradual incrementing of the spatial offset leads to a rapid diminishment of the top layer signal (R) relative to that of the second layer (W) in line with model's predictions. At a spatial offset $>40 \mu \mathrm{m}$ one already begins to see the bands of the third layer (Y) which keeps increasing relative to the intensity of the Raman bands of both the top (R) and second (W) layers.

The experiments were carried out again on the same sample using the defocusing (DLR) method. Again a similar pattern is seen (see Fig. 5b). The top layer signal (R) diminishes in relative terms to the second layer signal (W) with increasing displacement of the sample from the micro- scope objective. However, in this case, the rate of the change is much smaller and the ultimate ratio reached at $1 \mathrm{~mm}$ is much lower than that with the SO method at $160 \mu \mathrm{m}$ offset. In addition, the third (Y) layer is only tenuously resolved at the extreme displacement of $1 \mathrm{~mm}$.

Overall, the fully separated illumination-collection point (SO) variant outperformed the defocusing (DLR) configuration by a factor of $\times 14$. The comparison study enables Raman spectroscopists to evaluate a specific application on existing confocal Raman microscopy systems with defocusing micro-SORS and then project expected performance with a more complex fully spatially offset design requiring dedicated instrumentation. This would enable the end user to assess whether extra resources required for customisation of the Raman microscope to permit a more advanced variant are warranted by the specific application. A possible implementation of the fully spatially offset method comprises bringing the laser beam on the sample surface via a separate optical route bypassing the Raman collection microscope ${ }^{16}$ or alternatively by spatially resolving images on the CCD detector in a standard confocal Raman microscope as demonstrated by Di et al. ${ }^{20}$ Although, the latter concept restricts the range of spatial offsets deployable by the field of view of the microscope.

In addition to establishing the chemical makeup of individual layers the micro-SORS technique is also capable of determining the order in which the layers are laid. This is achieved by examining the relative rate of the decay of signals from individual layers. ${ }^{17}$ It is also possible to determine whether two chemical components detected by micro-SORS belong to two separate layers or if they are deposited within a mixed up single-layer. ${ }^{23}$

As the defocusing micro-SORS utilises instrumentation identical to that used with confocal Raman microscopy it is
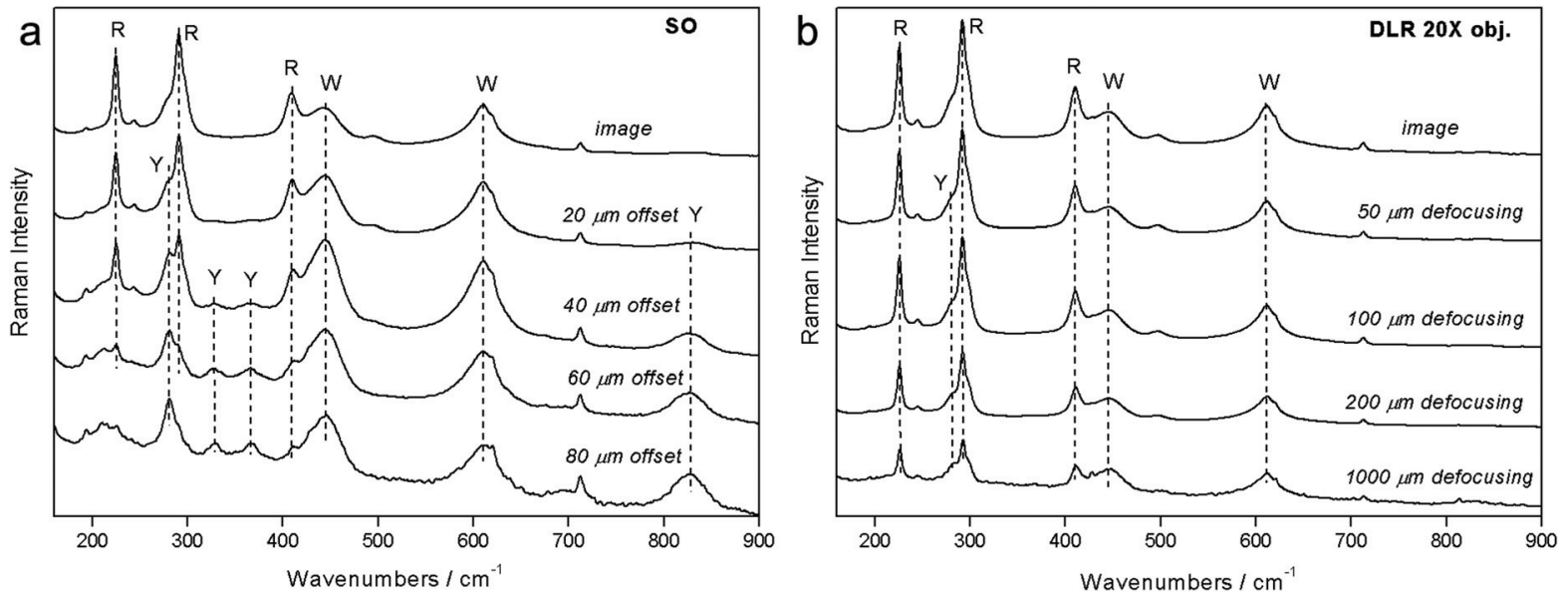

Fig. 5 Experimental results of micro-SORS measurements on a system consisting of two uppermost thin layers of paint (R - red (top layer), W white ( $1^{\text {st }}$ sublayer)) located on an extended substrate paint layer ( $Y$ - yellow ( $2^{\text {nd }}$ sublayer)) with (a) micro-SORS with full spatial separation between collection and illumination zones and (the spatial offset is indicated next to the spectra) and (b) defocusing micro-SORS (the sample displacement from the microscope objective is indicated next to the spectra). ${ }^{16}$ Adapted from C. Conti, C. Colombo, M. Realini, P. Matousek, Comparison of Key Modalities of Micro-scale Spatially Offset Raman Spectroscopy, Analyst, in press, DOI: 10.1039/c5an01900a, with permission of the Royal Society of Chemistry. 
important to understand which measurement type should be performed and which data interpretation method should be applied for a particular sample, as discussed by Conti et al. ${ }^{24}$ The distinction between the two methods of micro-SORS and conventional confocal Raman microscopy (CRM) is governed principally by the turbidity of the sample; when the sample is transparent on the scale of the $z$-scan ( $z$-scan magnitude « photon transport path length in the sample) the CRM interpretation of data is relevant and when the sample is diffusely scattering on the scale of the $z$-scan the micro-SORS interpretation applies.

It is also worth noting that if micro-SORS is performed by moving the sample from the original 'imaged' plane away from the microscope objective, as suggested above, the microSORS data cannot be confused with those of conventional Raman microscopy. This is because transparent samples would not be expected to produce major Raman signals in this $z$-scan region. However, if measurement is performed on a highly turbid sample (on the scale of the $z$-scans performed) by moving the microscope objective closer to the sample from the 'imaged' plane and applying the CRM interpretation, then entirely incorrect information on sample constitution would be derived as very different underlying photon propagation mechanisms are involved. To avoid this ambiguity in the CRM measurements a simple test is proposed ${ }^{24}$ to indicate which data interpretation should be applied when performing a $z$ scan on the sample whilst moving the microscope objective towards the sample from its initial sample surface 'imaged' position. The test consists of performing a $z$-scan in both the positive and negative directions away from the initial sample surface position. The symmetry or asymmetry of the obtained Raman intensities around the initial 'imaged' position then suggests whether the micro-SORS data interpretation should be applied or the CRM one; the symmetric response indicates a turbid sample and micro-SORS interpretation (unless a very thin transparent sample, on the scale of the depth resolution of Raman microscope, is present) whilst an asymmetric response suggests a transparent sample and a need to invoke CRM interpretation to the data.

\section{Emerging application areas}

Although the micro-SORS technique has been developed only very recently it has already been demonstrated conceptually in several potential application areas. Apart from validating the micro-SORS concept Fig. 3 and 4 also depict one of its important potential application areas - the non-invasive analysis of stratified polymers and the capability of the technique to isolate individual layers from each other. Multiple layers are often used in practical assemblies to achieve desirable properties of the final product - for example in food packaging the container needs to maintain certain mechanical properties and durability whilst it must not interact chemically with the content and provide an effective chemical barrier from the external environment. The analysis of these layers is important in many areas including polymer research and quality control in manufacture. However, these layers are often highly diffusely scattering and beyond the reach of conventional Raman microscopy and as such requiring cross sectional analysis - in this context micro-SORS represents an attractive non-invasive analytical alternative.

The capability of the method has also been demonstrated in isolating the chemical signature of different layers of paint with both artificially assembled systems ${ }^{2}$ and objects of art. ${ }^{3}$ The latter included painted sculptures and plasters. Fig. 6 exemplifies the use of micro-SORS on the subsurface analysis of a red mantle of a sculpture of Christ's disciple originating from a prestigious devotional place called 'Varese Sacred Mount' (United Nations Educational, Scientific and Cultural Organization World Heritage site). The sculpture was repainted many times over the centuries and consequently stratigraphy comprises a number of red layers consisting of red pigments such as red lake, red lead, red ochre and cinnabar. The micro-SORS Raman spectra permit the identification of individual layers and their chemical nature. The 'imaged' position spectrum exhibits only red lead Raman bands, but with the increase of the defocusing distance, the relative contribution of cinnabar increases together with lead white, frequently used as an extender of the cinnabar pigment. The findings were substantiated independently by cross sectional Raman analysis.

Micro-SORS was also applied to the analysis of biological samples. It was used in a study of chemical composition of cortical and cancellous bone samples ${ }^{20}$ and seeds. ${ }^{17}$ Fig. 7 demonstrates its use in the analysis of wheat seed. Despite high turbidity of the outer shell of the seed which also dominates the signals of conventional confocal Raman microscopy, micro-SORS permitted to separate the inner content (starch) from its outer shell Raman signatures. The observations were consistent with the results of independent conventional cross sectional Raman analysis. Grain composition is an important quality marker in food processing as the composition can affect the quality of the grains, and linking the seed composition as well as seed development during processing and storage with the end quality is of major importance. In addition, the identification of diseases at an early stage is of significant importance for grain producers, which calls for rapid and non-destructive methods for characterisation of inner grain components. Micro-SORS as a non-destructive technique, in order to rapidly characterise chemical components within a grain quantitatively or to monitor anomalies, thus holds a great potential in this area.

Conti et al. has also demonstrated the potential of microSORS in the area of non-invasive analysis of internal composition of processed paper, ${ }^{17}$ another important analytical area with potential application in manufacture quality control. The micro-SORS approach was shown to be capable of resolving non-invasively thin layers within a glossy paper revealing the presence of distinct layers within the sample; a top layer of rutile $\left(\mathrm{TiO}_{2}\right)$, a layer of calcite $\left(\mathrm{CaCO}_{3}\right)$ below and a layer of pure cellulose underneath within the body of the paper. 

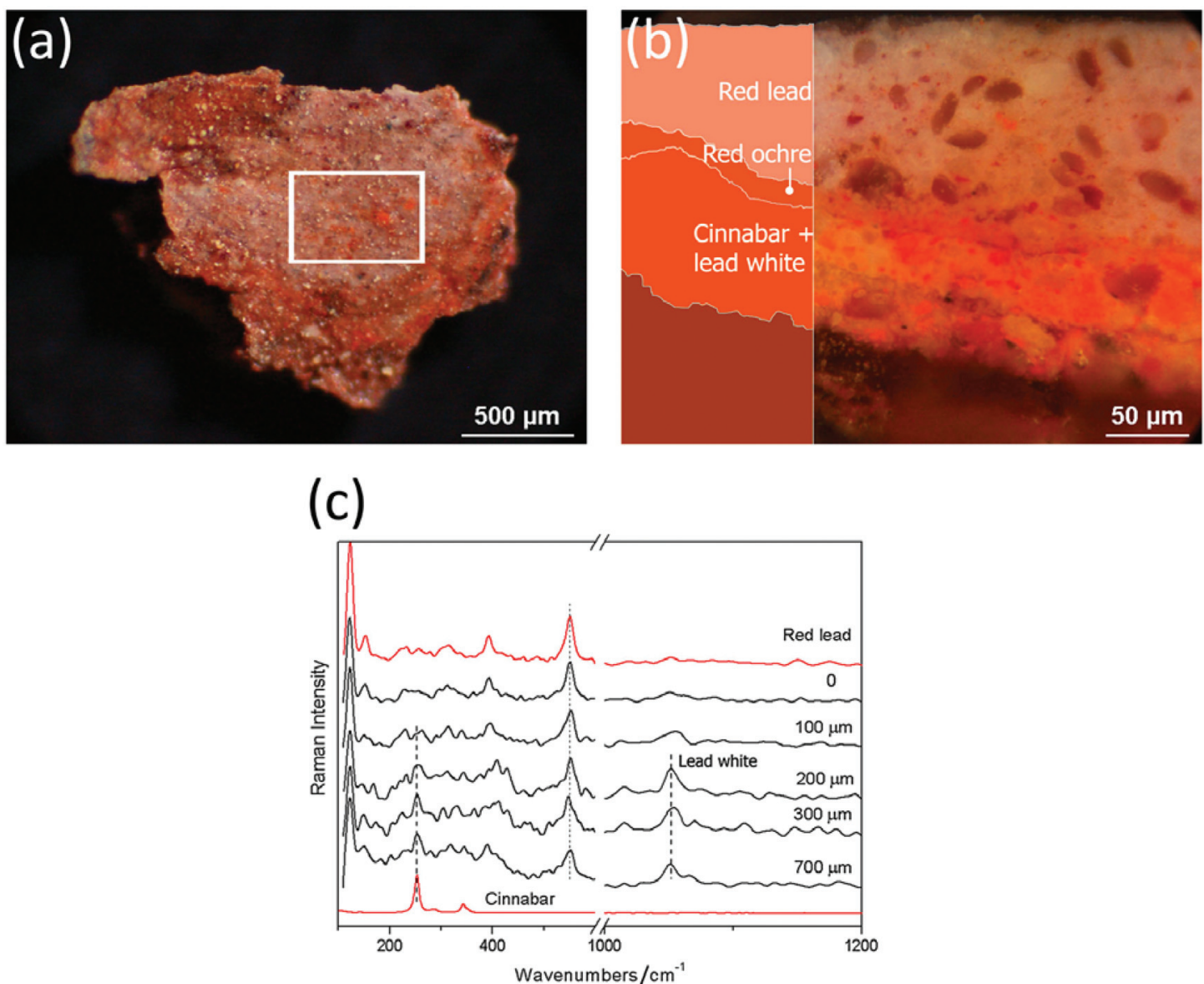

Fig. 6 Micro-SORS on a painted sculpture of Christ's disciple from Varese Sacred Mount in Northern Italy: (a) fragment image (the white square indicates the area analysed with micro-spatially offset Raman spectroscopy); (b) optical image and scheme of the stratigraphy; and (c) the defocused spectra are shown for different distances from the imaged plane indicated next to each spectrum $(0=$ 'imaged' position). The spectra are offset for clarity. Note that the line markers are for guidance to emphasise the changing relative intensity of the red lead, cinnabar and lead white with defocusing. The reference spectra are acquired on the sample cross section using conventional Raman spectroscopy. ${ }^{3}$
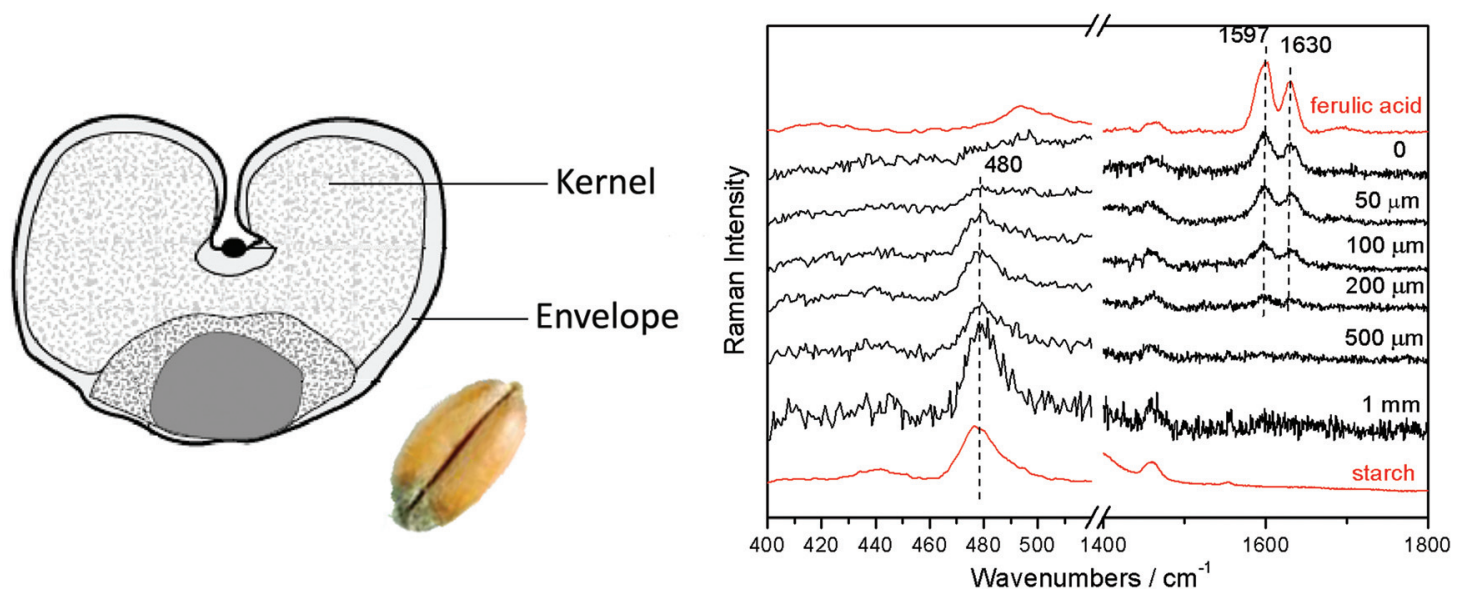

Fig. 7 Defocused micro-SORS spectra of a wheat seed ("Olivin" variety, a winter wheat variety grown in Norway) shown for different distances from the "imaged" plane indicated next to each spectrum ( $\Delta z=0$, "imaged" position). The spectra are offset for clarity. Note the line markers are for guidance to emphasize the changing relative intensity of ferulic acid and starch with the defocusing distance; the reference spectra, shown in red, were acquired on the sample cross section using conventional Raman spectroscopy. ${ }^{17}$ 
Overall, given the wide applicability of the technique and potential for further improvements by introducing fully separated illumination and collection micro-SORS modalities the concept is expected to find a number of application niches in several areas providing a complementary analytical capability to conventional Raman microscopy in situations where the accessible depths of conventional Raman microscopy are inadequate.

\section{Conclusions}

The recently proposed and demonstrated concept of microSORS combining traditional SORS concept with microscopy widens the applicability of conventional Raman microscopy by extending its accessible depths by up to an order of magnitude. As such thin multiple turbid layers of paint, biological interfaces, coatings and polymer layers can be analysed noninvasively at depths previously inaccessible by conventional Raman microscopy avoiding thus a need for invasive crosssectional analysis. This paves way for a host of new analytical applications across a range of disciplines.

\section{References}

1 T. Dieing, O. Hollricher and J. Toporski, Confocal Raman Microscopy, Springer, 2011.

2 C. Conti, C. Colombo, M. Realini, G. Zerbi and P. Matousek, Appl. Spectrosc., 2014, 68, 686-691.

3 C. Conti, M. Realini, C. Colombo and P. Matousek, J. Raman Spectrosc., 2015, 46, 476-483.

4 P. Matousek, I. P. Clark, E. R. C. Draper, M. D. Morris, A. E. Goodship, N. Everall, M. Towrie, W. F. Finney and A. W. Parker, Appl. Spectrosc., 2005, 59, 393-400.

5 K. Buckley and P. Matousek, Analyst, 2011, 136, 3039-3050.

6 B. B. Das, F. Liu and R. R. Alfano, Rep. Prog. Phys., 1997, 60, 227-292.

7 J. C. Hebden, A. Gibson, R. Yusof, N. Everdell, E. M. C. Hillman, D. T. Delpy, S. R. Arridge, T. Austin, J. H. Meek and J. S. Wyatt, Phys. Med. Biol., 2002, 47, 4155-4166.
8 H. Koizumi, Y. Yamashita, A. Maki, T. Yamamoto, Y. Ito, H. Itagaki and R. Kennan, J. Biomed. Opt., 1999, 4, 403413.

9 T. J. Pfefer, K. T. Schomacker, M. N. Ediger and N. S. Nishioka, Appl. Opt., 2002, 41, 4712-4721.

10 N. Ghosh, S. K. Majumder, H. S. Patel and P. K. Gupta, Opt. Lett., 2005, 30, 162-164.

11 L. Quan and N. Ramanujam, Opt. Lett., 2002, 27, 104-106.

12 S. C. Gebhart, S. K. Majumder and A. Mahadevan-Jansen, Appl. Opt., 2007, 46, 1343-1360.

13 C. Eliasson, M. Claybourn and P. Matousek, Appl. Spectrosc., 2007, 61, 1123-1127.

14 C. Eliasson, N. A. Macleod, L. C. Jayes, F. C. Clarke, S. V. Hammond, M. R. Smith and P. Matousek, J. Pharm. Biomed. Anal., 2008, 47, 221-229.

15 J. Hooijschuur, E. Ingeborg, I. Petterson, G. R. Davies, C. Gooijer and F. Ariese, J. Raman Spectrosc., 2013, 44, 1540-1547.

16 C. Conti, C. Colombo, M. Realini and P. Matousek, Analyst, 2015, 140, 8127-8133.

17 C. Conti, M. Realini, C. Colombo, K. Sowoidnich, N. K. Afseth, M. Bertasa, A. Botteon and P. Matousek, Anal. Chem., 2015, 87, 5810-5815.

18 A. Govil, D. M. Pallister and M. D. Morris, Appl. Spectrosc., 1991, 45, 1604-1606.

19 A. Govil, D. M. Pallister and M. D. Morris, Appl. Spectrosc., 1993, 47, 75-79.

20 Z. Di, B. H. Hokr, H. Cai, K. Wang, V. V. Yakovlev, A. V. Sokolov and M. O. Scully, J. Mod. Opt., 2014, 62, 97-101.

21 P. Matousek, C. Conti, C. Colombo and M. Realini, Appl. Spectrosc., 2015, 69, 1091-1095.

22 P. Matousek, M. D. Morris, N. Everall, I. P. Clark, M. Towrie, E. Draper, A. Goodship and A. W. Parker, Appl. Spectrosc., 2005, 59, 1485-1492.

23 C. Conti, M. Realini, A. Botteon, C. Colombo, S. Noll, S. R. Elliott and P. Matousek, Appl. Spectrosc., 2015, DOI: $10.1177 / 0003702815615345$, in press.

24 C. Conti, M. Realini, C. Colombo and P. Matousek, 2015, Contrasting Confocal with Defocusing Micro-Scale Spatially Offset Raman Spectroscopy, in press. 